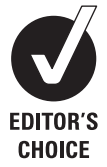

See editorial, page F392

'Department of Paediatric Cardiology, Royal Belfast Hospital for Sick Children, Belfast, UK

${ }^{2}$ Department of Radiology, Altnagelvin Area Hospital Derry, UK

${ }^{3}$ Centre for Public Health, The Queen's University of Belfast, Belfast, UK

\section{Correspondence to}

Dr Brian A McCrossan, Department of Paediatric

Cardiology, Royal Belfast Hospital for Sick Children, Falls Road, Belfast BT 12 6BE, UK; brianmccrossan@doctors. org.uk

Accepted 2 April 2011 Published Online First 17 May 2011

\title{
Fetal diagnosis of congenital heart disease by telemedicine
}

\author{
Brian A McCrossan, ${ }^{1}$ Andrew J Sands, ${ }^{1}$ Theresa Kileen, ${ }^{2}$ Chris R Cardwell, ${ }^{3}$ \\ Frank A Casey ${ }^{1}$
}

\section{INTRODUCTION}

Fetal diagnosis of congenital heart disease (CHD) varies across countries and regions. Prior to specific training schemes, most regions report a baseline detection rate of approximately $25 \% .{ }^{1-6}$ In the $\mathrm{UK}$, the best opportunity of screening for CHD is at the routine fetal anomaly ultrasound scan which is performed by radiographers with little experience and training in cardiac evaluation. ${ }^{1}$ Although training schemes have been shown to significantly improve prenatal detection of CHD, there is evidence that the beneficial effect diminishes with time. ${ }^{457}$ Effective screening for CHD may profit from additional strategies to provide ongoing support and opportunistic training for radiographers. Such schemes may partly compensate for a lack of exposure to CHD. ${ }^{8-11}$

Real-time fetal tele-echocardiography offers the possibility of earlier diagnosis of $\mathrm{CHD}$ and support for obstetric sonographers. The authors

\section{What is already known on this topic}

- Congenital heart disease (CHD) is accurately diagnosed and excluded in neonates by transmission of echocardiographic images across a telemedicine link.

- Transmitting fetal echocardiographic images, captured by a fetal cardiologist, across a real-time telemedicine link is feasible and accurate.

\section{What this study adds}

Fetal tele-echocardiography involving obstetric sonographers is feasible and reliable.

- CHD is accurately diagnosed and excluded in fetuses by transmission of echocardiographic images across a telemedicine link.

- Obstetric sonographers are very satisfied with and appreciate real-time fetal teleechocardiography facilitated by a fetal cardiologist.

hypothesised that telemedicine could assist in keeping patients close to home while also increasing access to specialist opinion. Tele-echocardiography in neonates has proved very successful in diagnosing and excluding CHD. ${ }^{12-18}$ The British Congenital Cardiac Association (BCCA) recommends that all tertiary congenital heart centres have telemedicine links with district general hospitals (DGHs) in their region. ${ }^{19}$ As fetal echocardiography is significantly different from trans-thoracic echocardiography ${ }^{1}$ 20 and no acceptable transmission bandwidth has been clearly established for conducting fetal teleechocardiography, a separate evaluation is necessary. The authors have published extensively on the role of telemedicine in paediatric cardiology and sought to extend this technology to fetal cardiology. 13 21-24

This study aimed to evaluate the feasibility, technical quality and diagnostic accuracy of fetal tele-echocardiography. It also sought to assess radiographers' opinions on the value of a fetal tele-cardiology service.

\section{METHODS}

The study was conducted at the Royal Belfast Hospital for Sick Children, and Altnagelvin 
Table 1 Study inclusion criteria

\begin{tabular}{ll}
\hline Fetal anomaly scan abnormality & Antenatal risk factor \\
\hline Suspected CHD on fetal anomaly scan & CHD in first-degree relative of fetus (requiring surgical or \\
& catheter intervention during first year of life) \\
Fetal dysrhythmia & Maternal IDDM \\
Hydrops fetalis & Exposure to teratogens associated with CHD (maternal or \\
& paternal)
\end{tabular}

Extra-cardiac fetal anomaly associated with CHD on fetal anomaly scan

CHD, congenital heart disease; IDDM, insulin-dependent diabetes mellitus.

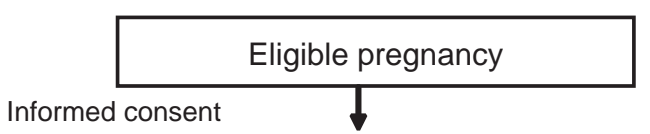

Diagnosis 1

\section{Diagnosis 2}

+ informed of provisional $\Delta$

Diagnosis 3

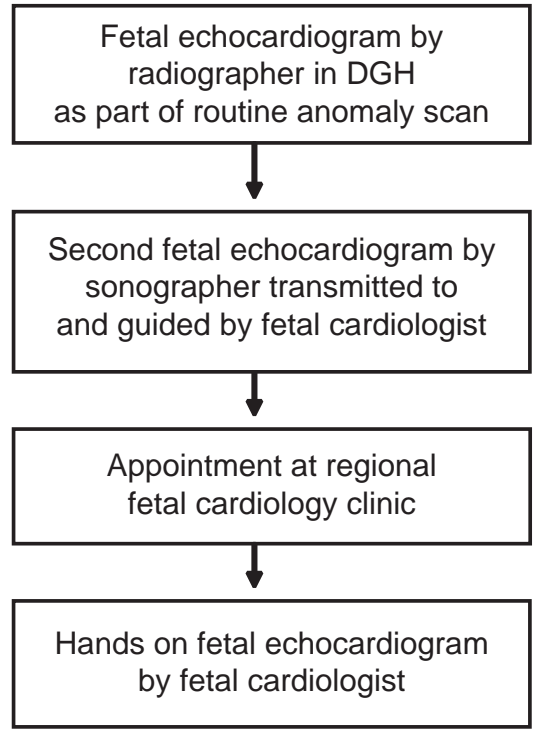

Figure 1 Study protocol. DGH, district general hospital.

Area Hospital (ALT). Ethical approval was obtained from the Central Office for Research Ethics Committees (COREC) and informed consent obtained from all study participants. Consecutive eligible patients were invited to participate in the study. Inclusion criteria mirrored the referral criteria to our fetal cardiology service (table 1).

The study protocol is summarised in figure 1 . Two sessions per week were potentially available for tele-links and additional slots were created for urgent cases. The tele-links were planned to coincide with the fetal anomaly scan when possible. Teleconferences were transmitted in real-time via ISDN6. The hands-on fetal echocardiogram (FE) is considered the reference standard. Following each FE, the parents were informed of the diagnosis, what future management was required and had the opportunity to ask questions.

FEs were performed using an ATL HDI 5000 ultrasound scanner (Advanced Technology Laboratories, Bothell, Washington, USA) which was replaced by a GE Voluson 730 4D (General Electric, Milwaukee, Wisconsin, USA) (at both ALT and regional unit). Both ultrasound scanners have a fetal cardiology package offering two-dimensional echocardiography, M-mode, pulsed wave and continuous wave Doppler and colour Doppler. At both sending and receiving sites, a Tandberg 880 (Tandberg, Lysaker, Norway) videoconferencing unit was employed.
Table 2 Radiographers' attitudes to fetal echocardiography and involvement in the Fetal Echocardiography Telemedicine Study

\begin{tabular}{lll}
\hline & \multicolumn{2}{l}{ Mean Likert score 1-5 } \\
\cline { 2 - 3 } & Start & End \\
\hline I feel that my FE technique is good & 2 & 3.8 \\
I feel confident in detecting congenital heart defects & 1.8 & 4 \\
I find the telemedicine equipment easy to use & 3.4 & 4.8 \\
I think the feedback I get with each tele-linked scan is & 4.2 & 4.8 \\
beneficial & & 1.6 \\
$\begin{array}{l}\text { I feel awkward performing a FE in front of the } \\
\text { paediatric cardiologist }\end{array}$ & 2.6 & 1.4 \\
I think that it would be better if the fetal echoes were \\
$\begin{array}{l}\text { saved on video and sent for interpretation rather than } \\
\text { a 'live' telemedicine consultation }\end{array}$
\end{tabular}

$\mathrm{FE}$, fetal echocardiogram.

\section{Radiographer questionnaire}

A structured Likert scale questionnaire was completed by the obstetric sonographers involved in this study. Questions related to confidence in fetal echocardiography and attitudes towards telemedicine consultations (table 2). The questionnaire was completed at the start and end of the study period.

\section{Statistical analysis}

Qualitative assessment was measured using a five-point Likert scale ( 5 , very good; 4 , good; 3 , average; 2 , poor; 1 , very poor) and again expressed as median (IOR). Diagnostic accuracy was calculated using a coefficient of agreement. Comparisons of tele-consultation quality and radiographers' attitudes were carried out using the appropriate non-parametric test. Statistical analysis was performed using Windows SPSS Version 14.0.

\section{RESULTS}

Over 20 months, 67 pregnant women were recruited to the study. No patients refused consent. The median age of the study group was 31.3 years (range $=17.1-40.8$ years, IOR 27.1-35.4 years). The median gestational age at the time of the tele-link was 23 weeks (range $=21-26$ weeks, IOR 23-25 weeks). The fetal tele-echocardiograms were performed at this relatively late gestational age because this is the time that fetal anomaly scans are routinely performed in Northern Ireland.

Sixty-nine fetal tele-echocardiograms were performed on 67 women during the study period. There was one set of twins and a fetal tele-echocardiogram was repeated on one patient. One patient did not attend for hands-on FE and this has been taken into consideration when evaluating diagnostic accuracy. The timing of the three FEs along with the feasibility and quality of fetal tele-echocardiograms are shown in table 3 .

In 50/69 (72\%) cases all 12 aspects of the FEs were confidently assessed from the transmitted images. At least 11/12 
Table 3 Feasibility and quality of remote fetal tele-echocardiograms

\begin{tabular}{ll}
\hline $\begin{array}{l}\text { Median interval between FE } 1 \text { and } 2 \\
\text { (range, IQR) }\end{array}$ & 0-days (0-25 days, $0-5$ days) \\
$\begin{array}{l}\text { Median interval between FE } 2 \text { and } 3 \\
\text { (range, IQR) }\end{array}$ & 22 days (2-80 days, $11.5-32.5$ days) \\
Mean duration of tele-link (range, SD) & $13.9 \mathrm{~min}$ (6-35 min, $6.1 \mathrm{~min})$ \\
Median video quality (IQR) & $4 / 5(3.5-4.5)$ \\
Median audio quality (IOR) & $4 / 5(3.5-4.5)$ \\
Median ease of use (IOR) & $4 / 5(4-4.5)$ \\
Median overall quality (IOR) & $4 / 5(3.6-4.5)$ \\
\hline
\end{tabular}

$\mathrm{FE}$, fetal echocardiogram.

Table 4 Frequency of confidently assessing individual aspects of fetal echocardiogram

\begin{tabular}{ll}
\hline & N (\%) \\
\hline Situs & $69(100)$ \\
Cardiac chambers & $69(100)$ \\
Atrio-ventricular valves & $68(99)$ \\
$1^{\circ}$ Atrial septum & $68(99)$ \\
Ventricular septum & $58(84)$ \\
Left ventricular outflow tract & $68(99)$ \\
Right ventricular outflow tract & $68(99)$ \\
Crossing over of great arteries & $69(100)$ \\
Aortic and pulmonary valves & $69(100)$ \\
Aortic arch & $69(100)$ \\
Ductal arch & $69(100)$ \\
Pulmonary venous connections & $58(84)$ \\
\hline
\end{tabular}

structures were confidently assessed in $94 \%$ of cases. The most difficult structures to confidently assess were the ventricular septum (84\% success) and pulmonary venous connections ( $84 \%$ success) table 4.

In $19 / 69(28 \%)$ cases the quality of the transmitted image was adversely affected by a range of factors relating to the fetal lie, obstructing limbs twin pregnancy and maternal obesity. Picture quality was rated as significantly lower in fetal teleechocardiograms affected by these problems (median Likert score $=3.5 / 5$ vs $4 / 5, p<0.01$ (Mann-Whitney $U$ test)).

Using the 'hands-on' FE as the reference standard, 58/69 (84\%) FEs demonstrated normal fetal hearts. In 11 (16\%) cases the FE was not normal with eight cases of major CHD including three duct-dependent lesions. Two patients had common arterial trunk, three had echogenic foci on the left atrio-ventricular valve. Critical aortic stenosis, Ebstein's anomaly, coarctation of aorta, double outright right ventricle, pulmonary atresia/ ventricular septal defect (VSD) and complete atrio-ventricular septal defect were diagnosed in one patient each.

\section{Diagnostic accuracy}

The initial FE (D1) agreed with the 'hands-on' FE in 46/68 (68\%) cases with disagreement in 22/68 (32\%). On 19 occasions CHD was incorrectly suspected and in three cases CHD was missed. These results indicate a fair level of agreement between the sonographer and fetal cardiologist $(\kappa$-statistic $=$ 0.25 , sensitivity $=0.70$, specificity $=0.67$ ).

In 56/68 (82\%) cases the fetal tele-echocardiogram (D2) indicated a normal fetal heart with 12/68 (18\%) suggesting CHD. On $97 \%$ of occasions D2 was confirmed by ‘hands-on' FE (D3). There was one false positive and one false negative diagnosis. In one fetal tele-echocardiogram a VSD was diagnosed. On the 'hands-on' FE again a VSD was suspected but on closer examination the ventricular septum was judged to be intact.
The false negative diagnosis was in a fetus with double outlet right ventricle. The fetus had multiple extra-cardiac abnormalities and the quality of the tele-link was noted to be poor because of maternal obesity and difficult fetal position. These results indicate an excellent level of agreement between fetal tele-echocardiography and 'hands-on' FE ( $\kappa$-statistic $=0.89$, sensitivity $=0.91$, specificity $=0.98$, positive predictive value $=$ 0.91 , negative predictive value $=0.98$ )

\section{Radiographer's evaluation}

Radiographers were highly satisfied with their involvement in the study. There was a consistent shift of responses towards increased confidence in fetal echocardiography technique and satisfaction with the telemedicine process (table 2 ). The mean total questionnaire score before the study was $16.8 / 30$ compared with $24.4 / 30$ after the study. This finding is confirmed on comparing individual radiographer's total questionnaire score (out of 30) at the start and end of the study. Using a Wilcoxon-signed ranks test there was a significant increase in questionnaire score following participation in this study $(p=0.043)$.

\section{DISCUSSION}

This study demonstrates that FE, performed by obstetric sonographers, facilitated by a telemedicine is technically feasible, reliable, diagnostically accurate and not particularly time consuming. This is the largest study of its kind. Sharma et al conducted a smaller, similar study. A high degree of diagnostic accuracy was also achieved. However, FEs were performed by paediatric cardiologists already proficient and a high proportion of tele-links were aborted (11\%). ${ }^{25}$ We believe the present study provides a more realistic assessment of the conditions under which a fetal tele-cardiology service would operate.

Fetal cardiology is well suited to telemedicine. It is a specialty principally involving medical imaging and is highly centralised. The vast majority of fetuses with CHD are born from pregnancies without a recognisable antenatal risk factor. ${ }^{1}$ Obviously, formal evaluation by a fetal cardiologist cannot be offered to every single pregnancy. ${ }^{4}$ In order to maximise detection of $\mathrm{CHD}$, greater proficiency from the non-specialist is required during the main formal evaluation of the fetus: the routine anomaly scan. This would be promoted by ongoing refreshment of skills. ${ }^{10}$ A significant positive feature of realtime fetal tele-echocardiography is the continued support provided to sonographers performing fetal anomaly scans in DGHs. ${ }^{7}$

A remote fetal cardiology service would have attendant costs which, unlike paediatric cardiology, are not offset by savings in interhospital transfers. ${ }^{21}$ The costs of delivering this service per centre comprise start-up and running costs. Start-up costs totalled $£ 6184$ (Codec and installation). The running costs are $£ 1895$ (line rental and service contract) and professional time and call costs $(6 \times$ standard phone call). However, the possible establishment of a 'one-stop-shop' for 'high-risk' pregnancies in which the routine fetal anomaly scan is followed, on the same day, by a remote FE could potentially reduce costs, relieve pressure on the regional service and present time and cost savings to the patient.

The relatively small study size and numbers with pathology (typical of this cohort) have reduced the power of the study and resulted in relatively wide CIs. The late gestational age at which the fetal tele-echocardiograms were performed could diminish their effectiveness by reducing the scope for 
intervention and termination. ${ }^{26}$ However, there is little reason to believe that fetal tele-echocardiography would not be as successful if performed between 18 and 20 weeks of gestation. ${ }^{27} 28$ Audio-visual contact with the parents is an undoubted advantage of real-time telemedicine. However, it is our experience that when CHD has been identified by fetal tele-echocardiography, parental counselling is more difficult. For example, conveying empathy across a tele-link to upset parents is difficult.

\section{CONCLUSIONS}

CHD may be reliably diagnosed and excluded by real-time telemedicine. It is highly acceptable to service users. Ultimately the aim of future work should be aimed towards the establishment of a regional fetal tele-echocardiography service that supports DGHs, improves access to fetal cardiology, reduces pressure on the regional services and reduces patient travelling and attendant costs.

Acknowledgements The authors would like to thank the obstetric sonographers and consultant obstetricians from Altnagelvin Area Hospital for their participation in this study. The authors would also like to acknowledge the advice provided by Sam McMaster of Questmark.

\section{Competing interests None.}

Funding Research was provided by the Royal Belfast Hospital for Sick Children.

Ethics approval This study was conducted with the approval of the COREC Peterborough committee.

Provenance and peer review Not commissioned; externally peer reviewed.

\section{REFERENCES}

1. Allan L. Antenatal diagnosis of heart disease. Heart 2000;83:367.

2. Garne E, Stoll C, Clementi M; Euroscan Group. Evaluation of prenatal diagnosis of congenital heart diseases by ultrasound: experience from 20 European registries. Ultrasound Obstet Gynecol 2001;17:386-91.

3. Friedberg MK, Silverman NH, Moon-Grady AJ, et al. Prenatal detection of congenital heart disease. J Pediatr 2009;155:26-31, 31.e1.

4. Hunter S, Heads A, Wyllie J, et al. Prenatal diagnosis of congenital heart disease in the northern region of England: benefits of a training programme for obstetric ultrasonographers. Heart 2000;84:294-8.

5. McBrien A, Sands A, Craig B, et al. Impact of a regional training program in fetal echocardiography for sonographers on the antenatal detection of major congenital heart disease. Ultrasound Obstet Gynecol 2010;36:279-84.

6. Bull C. Current and potential impact of fetal diagnosis on prevalence and spectrum of serious congenital heart disease at term in the UK. British Paediatric Cardiac Association. Lancet 1999;354:1242-7 ik.
7. Pézard P, Bonnemains L, Boussion F, et al. Influence of ultrasonographers training on prenatal diagnosis of congenital heart diseases: a 12-year population-based study. Prenat Diagn 2008;28:1016-22.

8. ter Heide H, Thomson JD, Wharton GA, et al. Poor sensitivity of routine fetal anomaly ultrasound screening for antenatal detection of atrioventricular septal defect. Heart 2004;90:916-17.

9. Cloutier A. Fetal echocardiography and telemedicine. Frontiers in Fetal Health 2001;3:191-3.

10. Allan L. Screening the fetal heart. Ultrasound Obstet Gynecol 2006;28:5-7.

11. Allan LD. How to establish a fetal cardiology service: experience in London and New York. Frontiers in Fetal Health 2001;3

12. Finley JP, Sharratt GP, Nanton MA, et al. Paediatric echocardiography by telemedicine - nine years' experience. J Telemed Telecare 1997;3:200-4.

13. Mulholland HC, Casey F, Brown D, et al. Application of a low cost telemedicine link to the diagnosis of neonatal congenital heart defects by remote consultation. Heart 1999;82:217-21.

14. Sable C. Telemedicine applications in pediatric cardiology. Minerva Pediatr 2003:55:1-13

15. Tsilimigaki A, Maraka S, Tsekoura T, et al. Eighteen months' experience with remote diagnosis, management and education in congenital heart disease. J Telemed Telecare 2001;7:239-43.

16. Widmer $\mathbf{S}$, Ghisla R, Ramelli GP, et al. Tele-echocardiography in paediatrics. Eur J Pediatr 2003:162:271-5.

17. Justo R, Smith AC, Williams M, et al. Paediatric telecardiology services in Queensland: a review of three years' experience. J Telemed Telecare 2004;10(Suppl 1):57-60.

18. Lewin M, Xu C, Jordan M, et al. Accuracy of paediatric echocardiographic transmission via telemedicine. J Telemed Telecare 2006;12:416-21.

19. Qureshi SA. Requirements for provision of outreach paediatric cardiology service. London: British Congenital Cardiac Association, 2008.

20. Gardiner HM. Fetal echocardiography: 20 years of progress. Heart 2001;86(Suppl 2):I112-22.

21. Grant B, Morgan GJ, McCrossan BA, et al. Remote diagnosis of congenital heart disease: the impact of telemedicine. Arch Dis Child 2010;95:276-80.

22. Casey FA. Telemedicine in paediatric cardiology. Arch Dis Child 1999;80:497-9.

23. McCrossan BA, Grant B, Morgan GJ, et al. Home support for children with complex congenital heart disease using videoconferencing via broadband: initial results. J Telemed Telecare 2008;14:140-2.

24. Morgan GJ, Craig B, Grant B, et al. Home videoconferencing for patients with severe congential heart disease following discharge. Congenit Heart Dis 2008;3:317-24.

25. Sharma S, Parness IA, Kamenir SA, et al. Screening fetal echocardiography by telemedicine: efficacy and community acceptance. J Am Soc Echocardiogr 2003;16:202-8.

26. Gardiner HM. Progression of fetal heart disease and rationale for fetal intracardiac interventions. Semin Fetal Neonatal Med 2005;10:578-85

27. Allan L, Dangel J, Fesslova V, et al.; Fetal Cardiology Working Group; Association for European Paediatric Cardiology. Recommendations for the practice of fetal cardiology in Europe. Cardiol Young 2004;14:109-14.

28. Rychik J, Ayres N, Cuneo B, et al. American Society of Echocardiography guidelines and standards for performance of the fetal echocardiogram. J Am Soc Echocardiogr 2004;17:803-10. 